\title{
Aero-Acoustic Performance of a Tube with Forward-Slanted Oblique Perforations for Reducing the Noise of a Supersonic Jet
}

\author{
Md. Tawhidul Islam Khan, Kunisato Seto ${ }^{\dagger}$, Zhixiang Xu and Mori Takeshi \\ Department of Mechanical Engineering, Saga University, Saga 840-8502, Japan
}

(Received 14 March 2003; accepted 20 January 2004)

\begin{abstract}
The aero-acoustic performance of a forward-slanted perforated tube attached to a convergent nozzle was evaluated experimentally. The sound pressure level and thrust of the tube were investigated and a good performance in noise suppression was found. Flow visualisation with the Schlieren system along with a high-speed video camera showed the disappearance of shock structure in the flow issued from the tube with forward-slanted perforations but not present in the flow from a solid tube without any perforations. The performance of the perforated tube with forward-slanted perforations was compared with that of different tubes 1) with backward-slanted perforations and 2) with perforations normal to the axis and 3) with a solid tube without any perforations. Among the tubes tested, the perforated tube with forward-slanted perforations showed the best performance. The tube suffered from minimum thrust loss compared with the backward- and the normally perforated tubes. The only a drawback of the tube with forward-slanted perforations was its generation of a tonal component at low pressure. The elimination of the inner sharp edge of the perforation or the reduction of tube thickness negated the drawback and improved the aero-acoustic performance of the tube with forward-slanted perforations.
\end{abstract}

${ }^{\dagger}$ Member of the International Institute of Acoustics and Vibration (IIAV)

\section{INTRODUCTION}

Most supersonic jets are imperfectly expanded. In these jets, a quasi-periodic shock cell structure is formed in the jet plume. The passage of a disturbance through the shock waves and the interaction of the shock waves with the disturbance generate intense shock-related noise in addition to turbulent mixing noise. There are two types of shock-related noise. One type has discrete spectral components, which are commonly referred to as jet screech tones. ${ }^{\mathbf{1 , 2}}$ The other type contains more broadband spectral components and is known as broadband shock-associated noise. ${ }^{3,4}$ The presence of the two kinds of shock-associated noise modifies the spectral and directional characteristics of the jet noise. The intensity of these noise components is a strong function of the direction of observation.

At high supercritical pressure ratios, the noise-generating mechanisms of the major sources of the radiated noise from supersonic jet flows (e.g., the mixing and the shock associated noise components) are often coupled. ${ }^{5}$ Normally, the intensity of the shock-generated acoustic radiation is directly dependent upon the shock strength and the level and coherence of the flow fluctuations convected through the shock front. $^{4,6}$ Therefore, to suppress aerodynamic noise components radiated by an imperfectly expanded supersonic jet, the extent, the spacing, and the strength of the repetitive shock structure and the level and coherence of the jet flow fluctuations convected through the shock fronts need to be modified, so that the overall strength of the noise-contributing sources and the effectiveness of their noise-generating mechanisms are reduced. Moreover, it is imperative that the useful modifications to the exhaust flows should be achieved with minimum thrust loss and weight penalty to the jet propulsion system.
Many devices, such as nozzles with tabs, slotted tubes, chuted nozzles, lobed nozzles, multi-tube nozzles, etc., are used for suppressing jet noise. Contoured and porous plug nozzles are also commonly used. ${ }^{7}$ However, in most cases the attachment of a noise-reducing device incurs undesirable effects, such as increasing dead weight and reducing thrust.

Supersonic jet-noise suppression achieved through the relief of the pressure of an under-expanded jet through a perforated tube has been reported by one of the present authors. In this paper the optimal length of the tube and the effects of the shroud on noise suppression have been described. ${ }^{\mathbf{8} 9}$ It has been demonstrated that the supersonic flow is generated by the addition of a perforated tube, and the flow at the exit of the perforated tube changes from under-expanded to correctly expanded or over-expanded jet as the length of the perforated tube is increased. Perforated tubes reduce noise most effectively when the optimum porosity, perforation pattern and length have been combined. The attachment of a shroud to a perforated tube, with proper design, not only reduces the energy loss but also improves the acoustical characteristics of the tube. ${ }^{10,11}$ In the present experimental study, a comparison of different types of perforations of the tube has been performed, and the forward-slanted perforation was found to be most favourable in suppressing supersonic jet noise.

\section{EXPERIMENTAL PROCEDURES}

\subsection{Nozzle, Perforated Tube and Jet Properties}

A convergent nozzle was used in the present experiment to generate an under-expanded supersonic jet of interest. Preliminary experiments were carried out to obtain the reference data for the nozzle. The convergence angle, exit diameter $(D)$, lip thickness, total length and the length of the converging parts of the nozzle were $30^{\circ}, 10,10,110$ and $26 \mathrm{~mm}$ re- 